\title{
Improvement in SNR with digital coding technique in OFDM transmission system
}

\author{
Shivnarayan Ahirwar, Abhishek Mishra \\ Department of Electronics and Communication Engineering, OIST Bhopal
}

\begin{abstract}
The paper describes the transmission of 32-QAM signals in an OFDM system with 128-point IFFT at transmitter and FFT at receiver. The channel over which transmission will take place is AWGN channel. Cyclic prefix for OFDM is calculated for improving bandwidth efficiency of the system. First, the symbol rate (SER) of coded OFDM system with transmission of BPSK signals in an OFDM system with 64-point IFFT at transmitter and FFT at receiver is plotted as a function of SNR via simulation in MATLAB and then the result is compared with coded OFDM system's SER plot, where the coding is provided by Trellis encoding at the transmitter accompanied by viterbi decoder at the receiver side.
\end{abstract}

Keywords: OFDM, ISI, Fading, SER, Cyclic Prefix, trellis encoding, viterbi decoder

\section{Introduction}

In the last years wireless communications have experienced a fast growth due to the high mobility that they allow. However, wireless channels have some disadvantages, like multipath fading, which results in severe degradation of reception performance [1], that make them difficult to deal with. Signal from transmitter not only takes a direct path or line of sight to reach destination but it gets reflected from different obstructions in channel and reaches destination through multiple paths. Thus, it experiences different phase shifts, time delays and attenuation that may result in constructive or destructive interference of the signal at the receiver. These delayed signals cause inter symbol interference at the receiver. It may also result in the failure of the communication link due to deep fade caused by frequency selective fading. A modulation that efficiently deals with selective fading channels is orthogonal frequency division multiplexing (OFDM) [2], which consists on $N$ orthogonal subcarriers generated and modulated in frequency domain.

The fact that the subcarriers are orthogonal allows the usage of the Fourier transform without introducing inter-carrier interference (ICI) [3] [4]. The advancements in digital signal processing and very large scale integrated circuits allow efficient and cost-effective implementation of the fast Fourier transform (FFT) operations making OFDM an attractive solution for wireless channels. Orthogonal frequency division multiplexing (OFDM) is one of the efficient techniques which is utilized to combat inter symbol interference, making the transmission over wireless channel less prone to frequency selective fading and thus improving the quality and reliability of wireless communication channel.[5] [6]

The symbol error rate performance at receiver can be improved by utilizing a channel coding scheme called Trellis coding [7] which provides robustness against channel impairments such as noise, interference and fading. At the receiver, viterbi decoding can be used to decode data in such a way as to minimize the probability of error by performing maximum likelihood decoding.

This paper is organized as follows. System model are presented in section 2. Calculation for length of cyclic prefix, simulation results for two different modulated coded and uncoded OFDM systems are presented in section 3 , followed by comparison in section 4 . Conclusion is provided in section 5 .

\section{Ofdm System}

The OFDM transceiver includes the following block for the transmission of message signal:



Fig.1 The OFDM transmitter system 


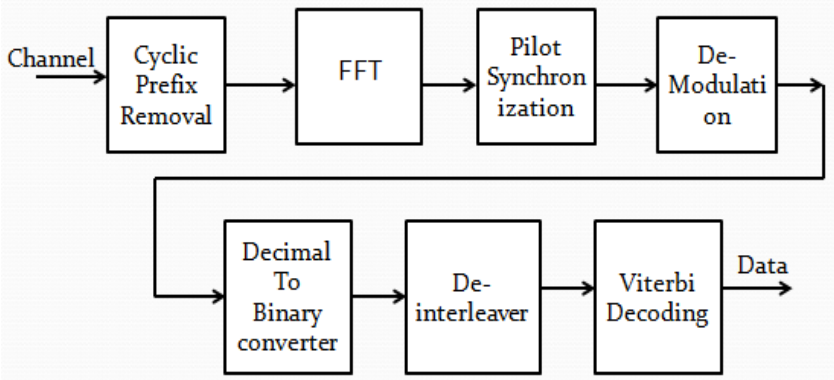

Fig.2 The OFDM receiver system

The main objective is to design an OFDM Transmitter and Receiver system with less BER. For this purpose the modulation technique and FFT/IFFT [8] are the main building blocks of OFDM. In our approach 32-QAM [9] and 128 point IFFT/FFT are used. But before describing those we are explaining some basic blocks of OFDM.

\subsection{Trellis Encoding}

A convolutional encoder [10] is a finite state machine. An encoder with $n$ binary cells will have $2 n$ states. An actual encoded sequence can be represented as a path on this graph. One valid path is shown in red as an example. This diagram gives us an idea about decoding: if a received sequence doesn't fit this graph, then it was received with errors, and we must choose the nearest correct (fitting the graph) sequence.

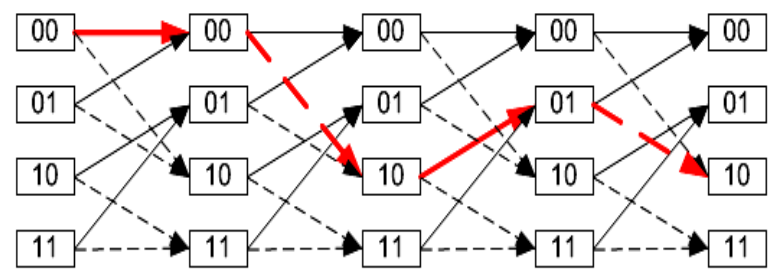

Fig 3. Trellis Structure

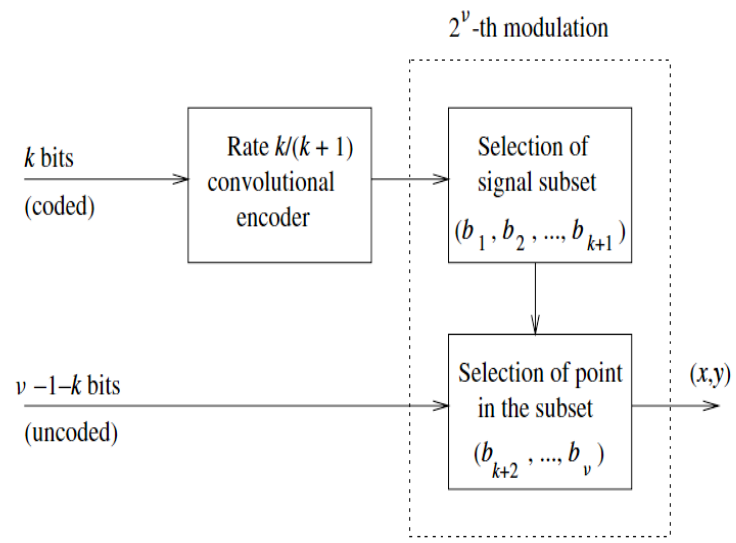

Fig 4 General encoder of rate(v-1)/v trellis code

\subsection{Viterbi Detector}

A Viterbi decoder uses the Viterbi algorithm for decoding a bitstream that has been encoded using forward error correction based on a convolution code. This is a maximum

likelihood decoder in the sense that it finds the closest coded sequence ${ }^{-} v$ to the received sequence ${ }^{-} r$ by processing the sequences on an information bit-by-bit (branches of the trellis) basis. In other words, instead of keeping a score of each possible coded sequence, the $V D$

tracks the states of the trellis.There are other algorithms for decoding a convolutionally encoded stream (for example, the Fano algorithm). The Viterbi algorithm [11] is the most resource-consuming, but it does the maximum likelihood decoding. It is most often used for decoding convolutional codes with constraint lengths $\mathrm{k}<=10$, but values up to $\mathrm{k}=15$ are used in practice. 


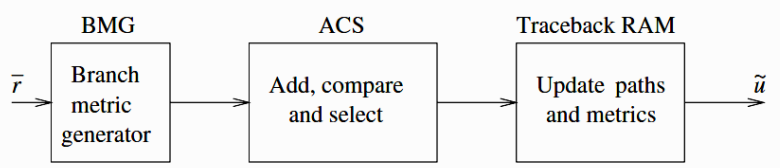

Fig 5 Block Diagram of Veterbi Decoder

\subsection{Quadrature Amplitude Modulation}

QAM is a signal in which two carriers shifted in phase by 90 degrees are modulated and the resultant output consists of both amplitude and phase variations. The number of points on the constellation is indicated in the modulation format description, e.g. 32QAM uses a 32 point constellation.

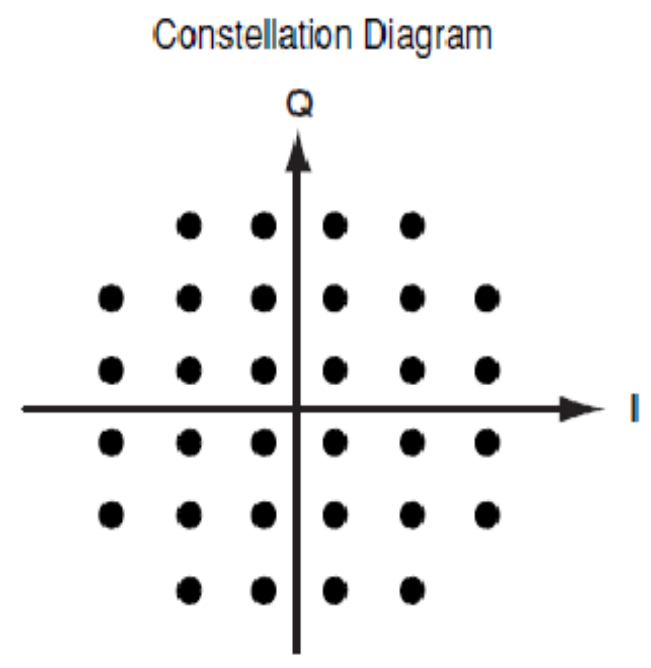

Fig 6. 32-QAM mapping

More points on the constellation, it is possible to transmit more bits per symbol. However the points are closer together and they are therefore more susceptible to noise and data errors.

In this case there are six $I$ values and six $Q$ values resulting in a total of 36 possible states $(6 \times 6=36)$. This is too many states for a power of two (the closest power of two is 32 ).

So the four corner symbol states, which take the most power to transmit, are omitted. This reduces the amount of peak power, the transmitter has to generate. Since $2^{5}=32$, there are five bits per symbol and the symbol rate is one fifth of the bit rate.

\subsection{FFT/IFFT Blocks}

The OFDM signal is generated by implementing the Inverse Fast Fourier Transform (IFFT) at the transmitter and Fast Fourier Transform (FFT) at the receiver side. Waveforms which are analog in nature must be sampled at discrete points before the FFT/IFFT algorithm can be applied.

The DFT operates on sample time domain signal which is periodic. The equation for DFT is -

$$
\mathrm{X}(\mathrm{k})=\sum_{\mathrm{n}=0}^{\mathrm{N}-1} \mathrm{x}(\mathrm{n}) \mathrm{W}_{\mathrm{N}}^{\mathrm{nk}} \quad \text { for } \mathrm{K}=0,1, \ldots \ldots . \mathrm{N}-1
$$

On the other hand, the inverse DFT for data sequence $\{\mathrm{X}(\mathrm{k})\}$ with $\mathrm{k}=0,1,2 \ldots \ldots \mathrm{N}-1$ defined in equation (2).

$$
x(n)=1 / N \sum_{k=0}^{N-1} X(k) W_{N}^{-n k} \text { for } n=0,1, \ldots \ldots . N-1
$$

The computation of each point of DFT requires the (N-1) complex multiplication, (N-1) complex addition. Thus to compute $\mathrm{N}$ points in DFT require $\mathrm{N}(\mathrm{N}-1)$ complex multiplication and $\mathrm{N}(\mathrm{N}-1)$ complex addition. 


\subsection{8-Points Butterfly Diagram}

Higher radix FFT algorithm has less number of the nontrivial complex multiplications, compared with the radix-2 FFT algorithm which is the simplest form in all FFT algorithms [8]. In an example for 128-point FFT and 64-point FFT, the number of nontrivial complex multiplications of radix-8 FFT algorithm is 152 and 48 , which is only $58.9 \%$ and $48.9 \%$ of that of radix -2 FFT algorithm.

Thus, in order to save power dissipation of the complex multiplier operation we use 128 IFFT/FFT point.

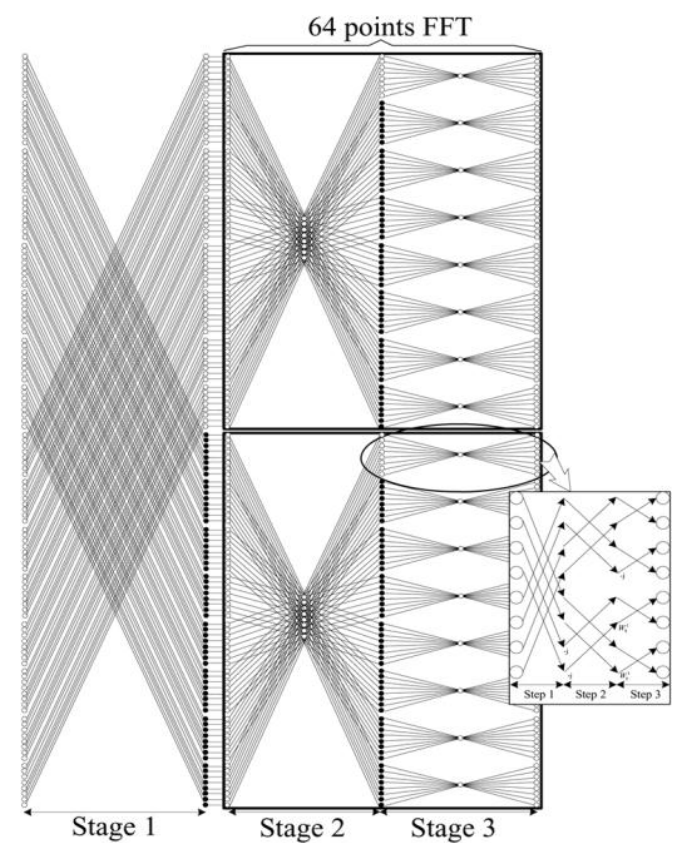

Fig 7. SFG of 128-point mixed-radix FFT

\section{Implemented Algorithm}

\subsection{At Transmitter end}

- Generate the Data "D".

- While D!=Null

\{

- Encode the generated data.

- Insert the interleaving Bits into Data.

- 32- QAM Mapping of data.

- Insert the Pilot Carrier

- Conversion into 128 IFFT Domain

\}

- Append Cyclic Prefix.

Transmit the generated data through AWGN channel 3.2 At Receiver end

- Receive the Data Bits "T".

- While T!=Null

\{

- Remove Cyclic Prefix

- Conversion into 128 FFT Domain

- Synchronize the Pilot Carrier.

- 32 QAM De-Modulation of received data.

- De-interleaving Bits from Data.

\}

- Decode the generated data.

Calculate the Bit Error Rate for the received Data. 


\section{Result}

The complete model for this OFDM transceiver with 32-QAM and 128 IFFT/FFT point is designed in the communication tool box in MATLAB 7.8.0

- We randomly generate the data using "randint" function provided in MATLAB for the random generation of data.

- Encoding of data is carried out by "Trellis Encoding".

- Insert the Interleaving Bits by using "matintrlv" function.

- QAM modulation is done of 32 QAM.

- After the cyclic prefix inserted data, we designed a channel for transmission, channel is prepared using "awgn" function.

- At the receiver side for the decoding purpose "Viterbi Detector " is used.

- Bit Error Rate is calculated using the formula:

- $\mathrm{BER}=$ Error Bits $/$ Length of Data

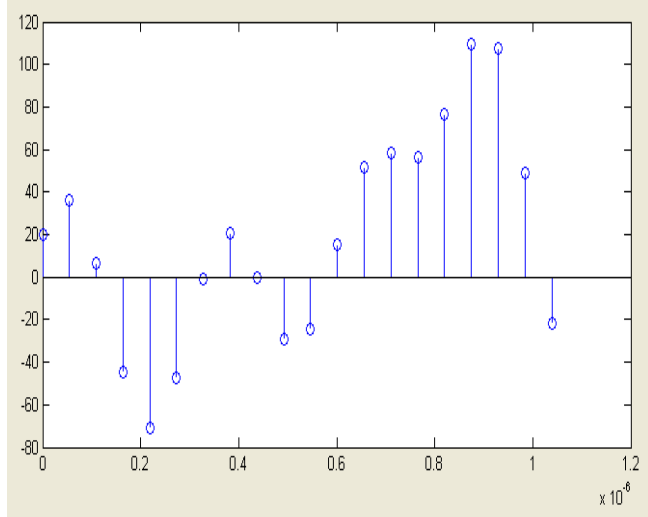

Fig 8. Randomly generated Data

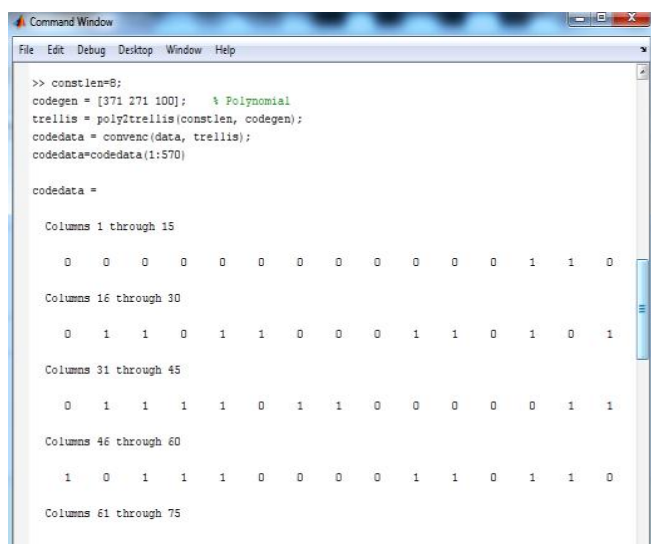

Fig 9. Encoding using Trellis Structure

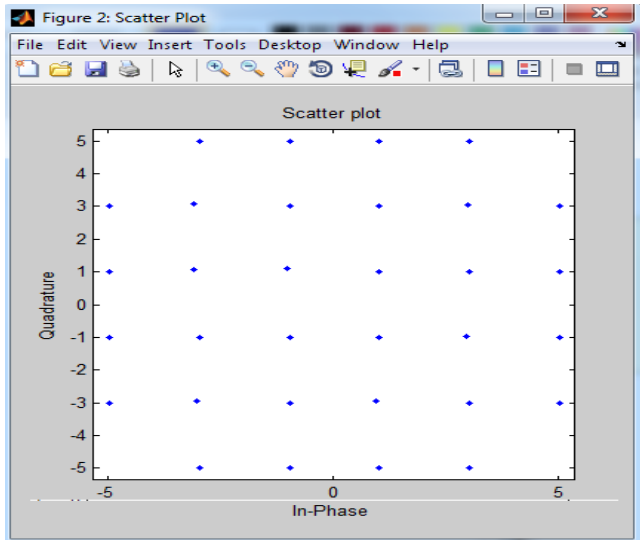

Fig 10. 32-QAM mapping 


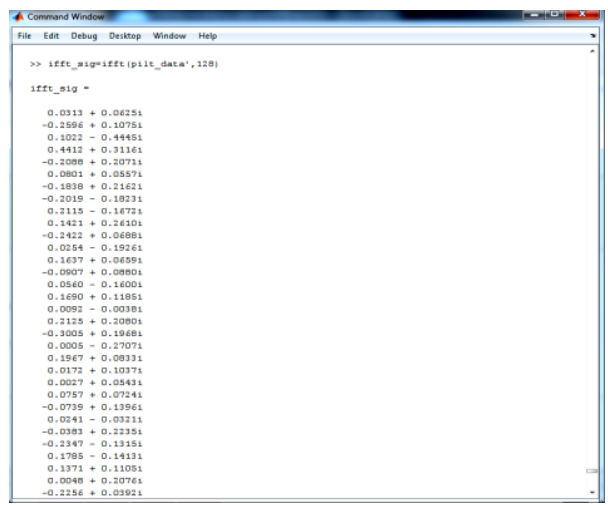

Fig 11. IFFT block Output

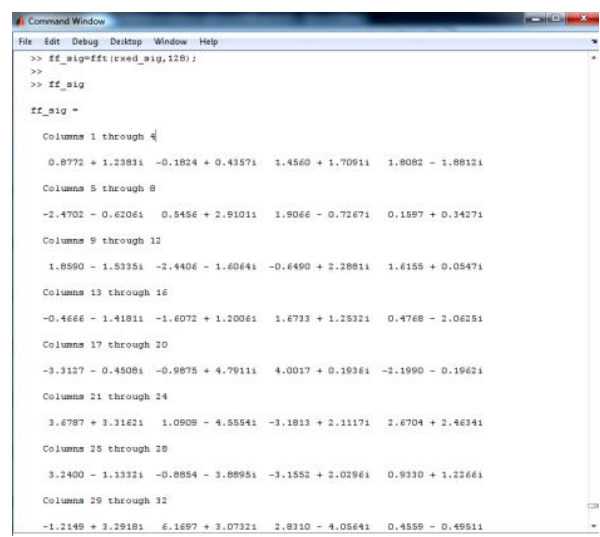

Fig 12. FFT block Output

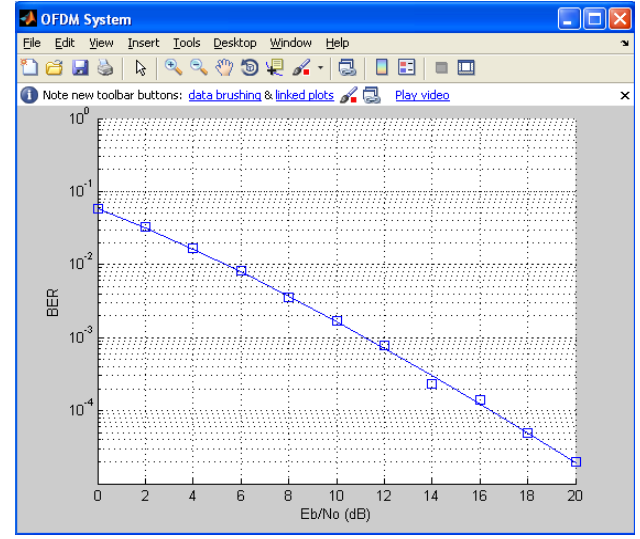

Fig 13. Plot between BER and SNR of proposed approach

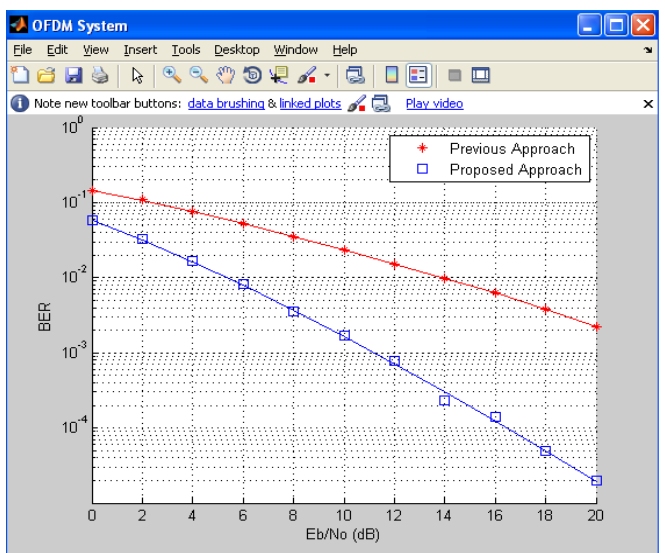

Fig 14. Comparative Result

www.iosrjournals.org 


\section{Conclusion}

OFDM is an efficient technique in mitigating the effect of ISI.This paper has proposed a scheme for BER reduction and improvement in SNR using 32 QAM and 128 point IFFT/FFT. The performance of 32 QAM signal in an OFDM system is compared to the one with an added feature of Trellis encoding. It is quite evident from the results that there is a significant improvement in performance if Trellis coding is used before transmission.The result founded were better when compared with the previous scheme available.

\section{References}

[1] Wei Zhang, Xiang-Gei Xia, Khaled Ben Letaief; "Space Time /frequency coding for MIMO-OFDM in next generation broadband wireless systems", IEEE Wireless communication, vol. 14,No. 3 pp. 32-43, June 2007

[2] R Nee and R Prasad, "OFDM for Wireless Multimedia Communications", Norwell, MA: Artech House, 2000. Fesdds

[3] Hassan A: Ahmad, Ahmed Iyanda Sulyman, Hossam S.Hassanein, "BER Perfonnance of OFDM System with Channel Impairment", $9^{\text {th }}$ IEEE International Workshop on Wireless Local Networks (WLN 2009) Zurich, Switzerland; 20-230ctober 2009.

[4] Chi-Hsiao Yih, "Etlects of channel estimation error on the BER pertonnance of OFDM systems in multipath rayleigh fading channels", Vehicular Technology conference, Baltimore, MD, pp. 1 097-11 01, Sept 2007.

[5] J G. Proakis, Digital Communications, 3rd edition. New York: McGraw- Hill, 1995.

[6] Y H. Kim, "Pertonnance analysis of a coded OFDM system in timevarying multipath Rayleigh fading channels," IEEE Trans. Veh. Technol. , vol. 48, no. 5, pp. 1610-1615, Sept 1999.

[7] Adnan . T, Masood .A "Use of Convolution Coding for Improving SER Performance of OFDM System", ICCSN -2011 3 rd IEEE conference, pp40-43, may 2011.

[8] Yu-Wei Lin and Chen-Yi Lee, "Design of an FFT/IFFT Processor for MIMO OFDM Systems" in IEEE transaction on circuits and systems - I: regular papers, vol. 54, no. 4, april 2007.

[9] Runfeng Yang and R. Simon Sherratt, "Enhancing MB-OFDM Throughput with Dual Circular 32-QAM" in IEEE Transactions on Consumer Electronics, Vol. 54, No. 4, November 2008.

[10] Yi Hong and Zhao Yang Dong, "Performance Analysis of Space-Time Trellis Coded OFDM System" in World Academy of Science, Engineering and Technology 132006.

[11] Andrei Vityaev and Paul H. Siegel, Fellow," On Viterbi Detector Path Metric Differences" in IEEE Transactions on Communication, Vol. 46, No. 12, December 2002 